\title{
Electroweak and QCD aspects in vector boson plus jets associated production with CMS
}

\author{
Nadezda Chernyavskaya *† \\ Swiss Federal Institute of Technology in Zurich (ETHZ), Zurich, Switzerland \\ E-mail: nadezda.chernyavskaya@cern.ch
}

\begin{abstract}
Total and differential cross sections of vector bosons produced in association with jets are studied at center-of-mass energies $\sqrt{s}=8$ and $13 \mathrm{TeV}$ with the CMS experiment at the LHC. Differential distributions as function of a broad range of kinematic observables are measured and compared to the theoretical predictions. Final states with a vector boson and jets can also be used to study electroweak initiated processes, such as production of the $\mathrm{Z}$ boson accompanied by a pair of energetic jets with large invariant mass. The cross section of this electroweak process is measured and the additional hadronic activity of events in a signal-enriched region is studied within the expected rapidity gap region.
\end{abstract}

The European Physical Society Conference on High Energy Physics

5-12 July, 2017

Venice

${ }^{*}$ Speaker.

${ }^{\dagger}$ on behalf of the CMS Collaboration 


\section{Introduction}

The production of a vector boson in association with jets is an important process at the LHC as it provides a reference to both physics measurements and detector response. Measurements of production cross sections can serve as fundamental tests of perturbative quantum chromodynamics(pQCD) and help to better understand strong interactions. In addition, production of vector bosons in association with jets is an important background for many Standard Model(SM) processes (single top and top quark pair production, vector boson fusion, Higgs boson production) as well as for beyond the SM searches. It is therefore crucial to measure the production cross section as a function of various kinematic variables with the highest possible precision. Here differential cross section measurements of vector boson production in association with jets in proton-proton collisions at $\sqrt{s}=8 \mathrm{TeV}[1,3]$ and $13 \mathrm{TeV}[2,4]$ at the LHC is presented.

Final states with a vector boson and jets can also be used to study electroweak initiated processes, such as pure electroweak production of the $l l j j$ final state. This EWK Zjj process has a distinctive signature of a Vector Boson Fusion (VBF) process: two jets with large energy and pseudorapidity separation produced in association with the dilepton pair from a $\mathrm{Z}$ boson decay. The study of such processes is a part of a more general investigation of $\mathrm{VBF}$ and scattering processes in both SM and physics beyond the SM. Properties of the EWK Zjj production can be compared to the theoretical predictions once isolated from the backgrounds. In particular, a study of additional hadronic activity in a signal-enriched sample provides tests of the soft QCD modelling. The measurement of the electroweak production of two jets in association with a $\mathrm{Z}$ boson in proton-proton collisions at $\sqrt{s}=13 \mathrm{TeV}$ at the LHC is presented here [5].

\section{Measurement of differential cross sections of $\mathbf{V}+$ jets production}

\subsection{W + jets}

The $\mathrm{W}$ plus jets associated production cross section is measured differentially at center-ofmass energy of $\sqrt{s}=8 \mathrm{TeV}$ [1] using $19.6 \mathrm{fb}^{-1}$ of integrated luminosity data and at $\sqrt{s}=13 \mathrm{TeV}$ [2] using $2.5 \mathrm{fb}^{-1}$ of data. The $\mathrm{W}$ bosons are identified through their $W \rightarrow \mu v$ decay mode. Muons are required to have transverse momentum $p_{T}>25 \mathrm{GeV}$ and pseudo-rapidity $|\eta|<2.1$, jets are required to have $p_{T}>30 \mathrm{GeV}$ and rapidity $|\mathrm{y}|<2.4$ and be spatially separated from muons with $\Delta R>0.5$. Events containing additional muons with $p_{T}>15 \mathrm{GeV}$ are vetoed. Events are required to have transverse mass of muon and missing transverse energy $M_{T}>50 \mathrm{GeV}$.

The cross sections are presented after deconvoluting the detector effects by an unfolding procedure and carried out with respect to the exclusive and inclusive jet multiplicities, jet $p_{T}$, jet $|\eta|$, jet transverse momentum scalar sum $H_{T}$ and, for $8 \mathrm{TeV}$ measurement, also with respect to dijet invariant mass, $p_{T}$, rapidity difference and spatial separation $\Delta R$. The $8 \mathrm{TeV}$ measurements are compared to particle-level simulated predictions using MADGRAPH 5[6] + PYTHIA 6[7]; SHERPA 2[9] and MADGRAPH 5 AMC@NLO[8] + PYTHIA 8 with next-to-leading order (NLO) calculation; BLACKHAT + SHERPA [10] (NLO); and $N_{\text {jetti }}$ [11] with next-to-next-to-leading order(NNLO) accuracy. Overall good agreement is observed with NLO multileg prediction and NNLO for W + 1 jet production. SHERPA 2 slightly overestimates the cross section at high $H_{T}$. The $13 \mathrm{TeV}$ measurement are compared to MADGRAPH 5 + PYTHIA 6; MADGRAPH 5 AMC@NLO + 
PYTHIA 8 (multileg NLO) and $N_{\text {jetti }}$ NNLO. Good agreement with predictions is observed, but overall LO MADGRAPH $5+$ PYTHIA 6 slightly underestimates data on the observables. Cross sections as a function of $H_{T}$ are shown on Figure 1 for $8 \mathrm{TeV}$ (left) and $13 \mathrm{TeV}$ (right).
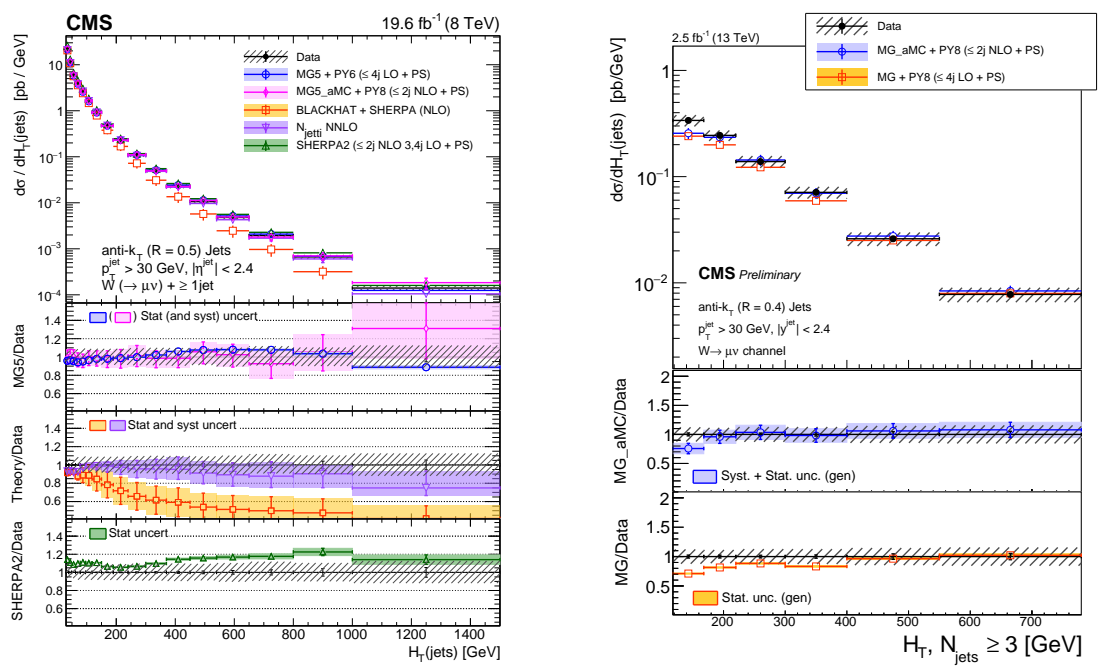

Figure 1: (right)The differential cross section measurement at (left) $\sqrt{s}=8 \mathrm{TeV}$ for $H_{T}$ for $N_{\text {jets }} \geq 1[1]$ and (right) $\sqrt{s}=13 \mathrm{TeV}$ for $H_{T}$ for $N_{\text {jets }} \geq 3$ [2], compared to the theoretical predictions. The lower plots show the ratio of each prediction to the unfolded data.

\section{$2.2 \mathrm{Z}+$ jets}

The $\mathrm{Z}$ plus jets associated production cross section is measured differentially at center-of-mass energy of $\sqrt{s}=8 \mathrm{TeV}$ [3] using $19.6 \mathrm{fb}^{-1}$ of integrated luminosity data and at $\sqrt{s}=13 \mathrm{TeV}$ [4] using $2.5 \mathrm{fb}^{-1}$ of data. The $\mathrm{Z}$ bosons are reconstructed using the $Z \rightarrow l l$ decay mode with either dimuons and, for $8 \mathrm{TeV}$ analysis, dielectrons. Leptons are required to have transverse momentum $p_{T}>20 \mathrm{GeV}$ and $|\eta|<2.4$, and the dilepton mass has to be within $20 \mathrm{GeV}$ of the nominal $\mathrm{Z}$ peak mass. Jets are required to have $p_{T}>30 \mathrm{GeV}$ and $|y|<2.4$ (for the extended multidimensional cross sections the phase space is extended to $|y|<4.7)$. Jets have to be spatially separated from both leptons of the $\mathrm{Z}$ boson decay with $\Delta R>0.4$.

The cross sections are obtained from the data distributions corrected to the particle level distributions using an unfolding procedure. The differential cross sections are measured as a function of numerous observables. Multidimensional differential cross sections are presented with the respect to up to three variables leading jet $p_{T}$, rapidity of the $\mathrm{Z}$ boson $y(Z)$ and the leading jet $y\left(j_{1}\right) .8 \mathrm{TeV}$ results are compared to particle-level simulated predictions using MADGRAPH 5 + PYTHIA 6; SHERPA 2 and MADGRAPH 5 AMC@NLO + PYTHIA 8 (NLO). The comparisons show very good agreement with the considered NLO multileg predictions and that it is essential to include a large number of final state partons in the matrix element to correctly describe the kinematics of the leading jets. On the Figure 2(left) the $d^{2} \sigma / d p_{T}\left(j_{1}\right) / d y\left(j_{1}\right)$ measurement is shown with the extended jet absolute rapidity range up to 4.7 . The dijet invariant mass is an important variable in the study of the vector boson fusion production processes. The measured cross section as a 
function of the dijet mass is shown on Figure 2(right). The predictions agree well with the measurement but discrepancies with LO and NLO predictions are observed at low mass region where the angle between the two jet directions is smaller than $\pi / 2.13 \mathrm{TeV}$ measurements are compared to predictions using MADGRAPH 5 AMC@NLO + PYTHIA 8 with NLO for 0,1 and 2 jets. The predictions generally describe the measured data well within the uncertainties.
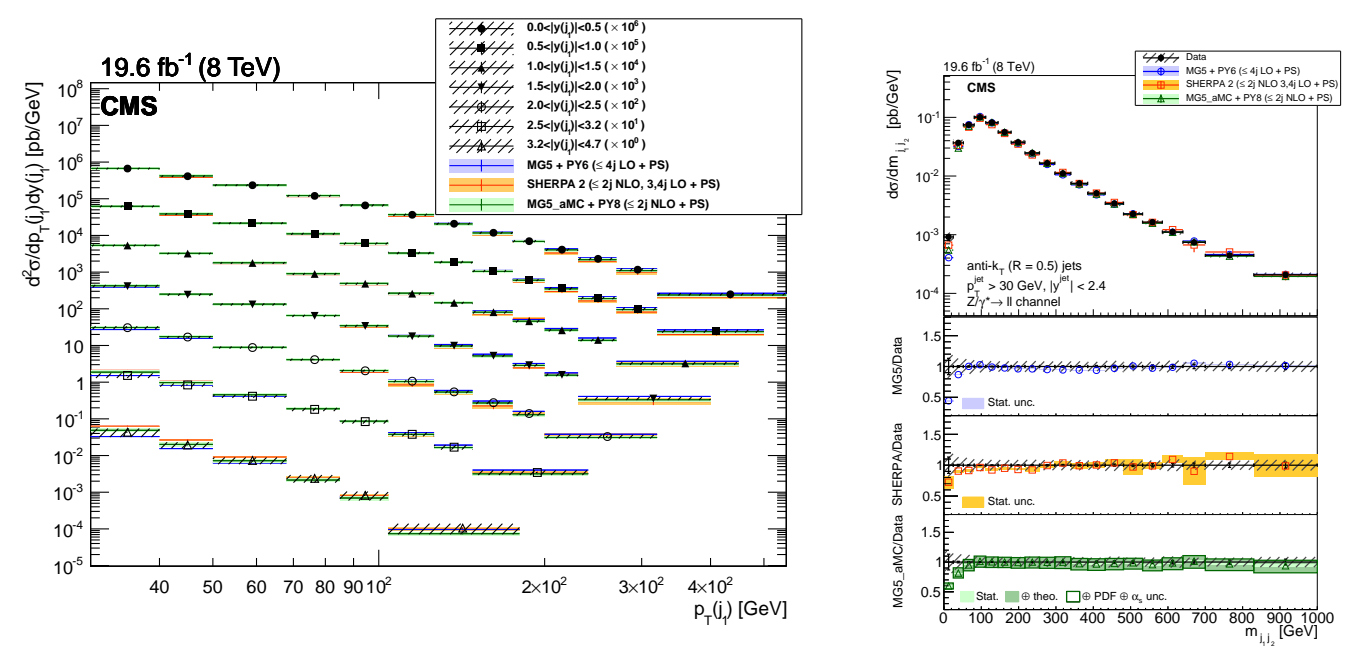

Figure 2: The differential cross section for $\mathrm{Z}(11)+$ jets production at $\sqrt{s}=8 \mathrm{TeV}$ as a function of (left)the leading jet $p_{T}, y$ and (right)dijet invariant mass for $N_{\text {jets }} \geq 2$. The bands around the measurement and prediction points represent the total uncertainties[3].

\section{Electroweak production of $Z+$ jets}

Measurement of the electroweak production of the $\mathrm{Z}$ boson in association with two jets is performed at $\sqrt{s}=13 \mathrm{TeV}$ with the integrated luminosity of $35.9 \mathrm{fb}^{-1}$. The signal is defined in the kinematic region with dilepton mass $M_{l l}>50 \mathrm{GeV}$, parton transverse momentum $p_{T}(j)>25$ $\mathrm{GeV}$, and diparton mass $M_{j j}>120 \mathrm{GeV}$, where the cross section of the lljj final state (with $1=\mathrm{e}$ or $\mu$ ) is expected to be $\sigma_{L O}(\mathrm{EWK} 1 \mathrm{jjj})=543+7$ (scale) $\pm 22(\mathrm{PDF}) \mathrm{fb}$. The main background in this topology is Drell-Yan(DY) plus jets events. For inclusive lljj final states background and signal diagrams with identical initial and final states can interfere which leads to a small interference that has to be taken into account when measuring the signal contribution.

Events are required to have at least two leptons(electrons or muons) with the leading lepton $p_{T}>30 \mathrm{GeV}$ and subleading $p_{T}>20 \mathrm{GeV},|\eta|<2.4$. The dilepton invariant mass has to be within $15 \mathrm{GeV}$ of the nominal $\mathrm{Z}$ boson mass. Events are required to have at least two jets with $p_{T}$ requirements on the leading and subleading jets $p_{T}\left(j_{1}\right)>50 \mathrm{GeV}, p_{T}\left(j_{2}\right)>30 \mathrm{GeV},|\eta|<4.7$, and dijet invariant mass $M_{j j}>200 \mathrm{GeV}$.

Electroweak signal is characterized by several distinct features like a large pseudorapidity separation between the tagging quark jets and large dijet invariant mass. In addition, the Zjj system is expected to be approximately balanced in the transverse plane which is not true for the background. 
Some other kinematic variables of the event and quark initiated jet properties can be used to separate signal from background. In order to enhance the separation these variables are used in the multivariate analysis and a boosted decision tree(BDT) is used to achieve an optimal separation. Figure 3(left) shows the distribution of the transformed BDT output for dimuon channel. Good agreement with data is observed.

A binned maximum likelihood fit is performed as a function of the BDT output. Nuisance parameters are used to constrain the background variation and introduce other systematics uncertainties. Dominant systematic effects on the signal determination are the jet energy scale variation and simulation statistics on the experimental side and the background QCD scales uncertainties on the theoretical side. The results obtained are compatible among the dilepton channels and the measured cross sections are the following : $\sigma($ EWK $\mu \mu \mathrm{jj})=540 \pm 23($ stat $) \pm 56$ (syst $) \mathrm{fb}$; $\sigma(\mathrm{EWK}$ eejj $)=554 \pm 34($ stat $) \pm 70($ syst $) f b ; \sigma($ EWK lljj $)=552 \pm 19($ stat $) \pm 55($ syst $) f b$ in agreement with the SM prediction.

Once the signal is established the properties of the additional hadronic activity in the expected rapidity gap between two VBF tagging jets are studied. In signal events a substantial suppression of the hadronic activity is expected because the final states originate form pure electroweak process in contrast to QCD radiative production of jets in the dominant DY Zjj background. To select a signal-enriched sample a BDT cut is applied BDT $>0.92$. To study additional jet activity jets with $p_{T}>15 \mathrm{GeV}$ are used. The $p_{T}$ of the $p_{T}$-leading additional jet and the scalar $p_{T}$ sum are studied and data are found to be in good agreement with expectations. To study additional charged hadronic activity a collection of high-purity tracks[12] with $p_{T}>0.3 \mathrm{GeV}$, uniquely associated with the main PV in the event, is formed. These tracks are then clustered in "soft track-jets" using anti- $\mathrm{k}_{T}$ clustering algorithm[13] with distance parameter of $\mathrm{R}=0.4$. The use of the track jets allows to reconstruct energy to as low as a few $\mathrm{GeV}$ and avoid any effect of pileup. Good agreement is observed between data and the simulation.

The efficiency of a gap activity veto corresponds to a fraction of events which do not have a rapidity gap activity above a given threshold. Measurements of veto efficiency in VBF-like topologies can quantify how reliable the modelling is in particular in a signal-enriched region. On the Figure 3(right) the veto efficiency of combined dilepton events in the signal enriched region with BDT $>0.92$ as a function of the applied threshold of the $p_{T}$ of the leading soft jet. Data are in a good agreement with the presence of the signal with two considered parton shower predictions PYTHIA and HERWIG. The data seem to prefer the signal model with HERWIG parton showering at low gap activity values while for larger gap activities PYTHIA description might be better.

\section{Summary}

Measurements of the cross sections and differential cross sections for a vector boson produced in association with jets at $\sqrt{s}=8$ and $13 \mathrm{TeV}$ are presented. Data are in good agreement with NLO and NNLO predictions. Electroweak production of the $\mathrm{Z}$ boson in association with two jets is studied and the cross section is measured with $10 \%$ precision. Validation of the rapidity gap activity modelling in VBF signal pure sample is performed by studying additional hadronic activity of events. 

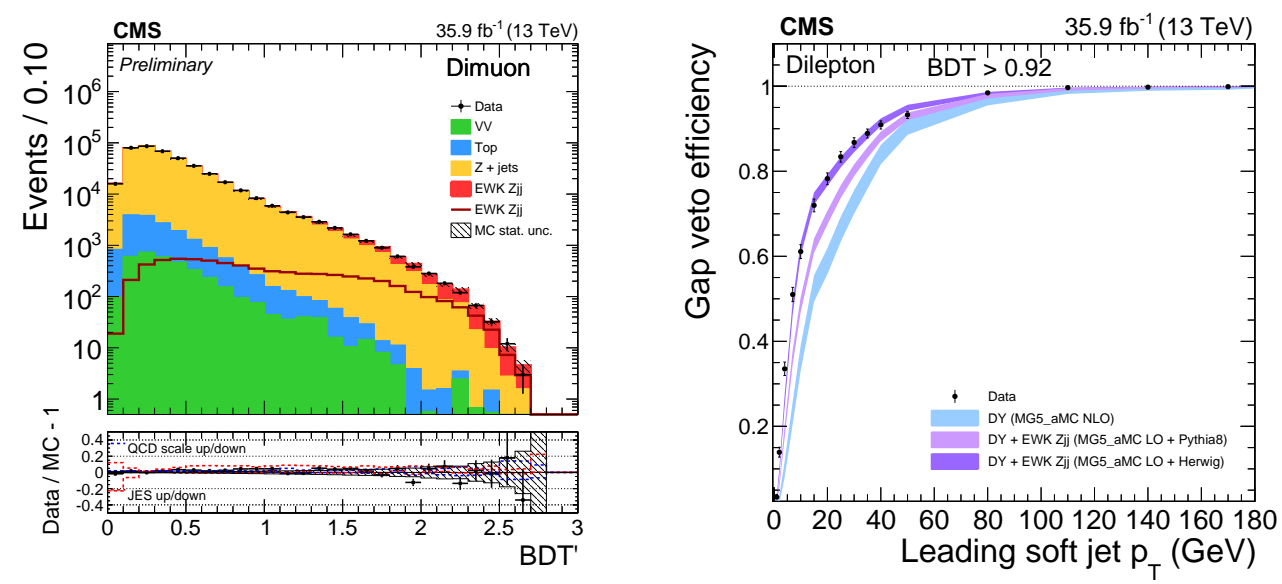

Figure 3: (left)Distributions for the BDT discriminants in dimuon events. The contributions from the different background sources and the signal are shown stacked, with data points superimposed. The lower panels show the relative difference between the data and expectations. (right) Efficiency of a gap activity veto in dielectron and dimuon events with BDT>0.92, as a function of the leading soft jet $p_{T}$. Data points are compared to MC expectations with only DY events, including signal the PYTHIAor the HERWIG ++ parton shower models. The bands represent the MC statistical uncertainty[5].

\section{References}

[1] V. Khachatryan et al. [CMS Collaboration], Phys. Rev. D 95 (2017) 052002 doi:10.1103/PhysRevD.95.052002 [arXiv:1610.04222 [hep-ex]].

[2] CMS Collaboration, CMS-PAS-SMP-16-005, http://cds.cern.ch/record/2204927.

[3] V. Khachatryan et al. [CMS Collaboration], JHEP 1704 (2017) 022 doi:10.1007/JHEP04(2017)022 [arXiv:1611.03844 [hep-ex]].

[4] CMS Collaboration, CMS-PAS-SMP-15-010, http://cds.cern.ch/record/2114819.

[5] CMS Collaboration, CMS-PAS-SMP-16-018, http://cds.cern.ch/record/2261499.

[6] J. Alwall, M. Herquet, F. Maltoni, O. Mattelaer and T. Stelzer, JHEP 1106 (2011) 128 doi:10.1007/JHEP06(2011)128 [arXiv:1106.0522 [hep-ph]].

[7] T. Sjostrand, S. Mrenna and P. Z. Skands, JHEP 0605 (2006) 026 doi:10.1088/1126-6708/2006/05/026 [hep-ph/0603175].

[8] J. Alwall et al., JHEP 1407 (2014) 079 doi:10.1007/JHEP07(2014)079 [arXiv:1405.0301 [hep-ph]].

[9] S. Hoeche, F. Krauss, M. Schonherr and F. Siegert, JHEP 1304 (2013) 027 doi:10.1007/JHEP04(2013)027 [arXiv:1207.5030 [hep-ph]].

[10] Z. Bern, L. J. Dixon, F. Febres Cordero, S. Hoeche, H. Ita, D. A. Kosower and D. Maitre, Comput. Phys. Commun. 185 (2014) 1443 doi:10.1016/j.cpc.2014.01.011 [arXiv:1310.7439 [hep-ph]].

[11] R. Boughezal, C. Focke, X. Liu and F. Petriello, Phys. Rev. Lett. 115 (2015) no.6, 062002 doi:10.1103/PhysRevLett.115.062002 [arXiv:1504.02131 [hep-ph]].

[12] CMS Collaboration, CMS-PAS-TRK-10-005, http://cds.cern.ch/record/1279383. 
[13] M. Cacciari, G. P. Salam and G. Soyez, JHEP 0804 (2008) 063 doi:10.1088/1126-6708/2008/04/063 [arXiv:0802.1189 [hep-ph]]. 